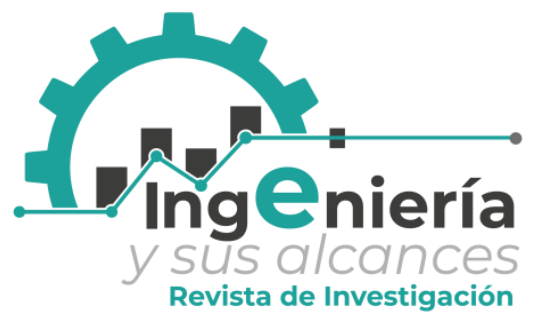

Ingeniería y sus alcances, Revista de Investigación Https://doi.org/10.33996/revistaingenieria.v4i10.66 Septiembre - diciembre 2020 Volumen 4 / No. 10 ISSN: $2664-8245$ ISSN-L: $2664-8245$

\title{
Aplicación web para fortalecer la gestión del transporte en la recolección de residuos sólidos urbanos
}

\author{
Web application to strengthen transport management in the collection of \\ urban solid waste
}

Aplicativo da web para fortalecer a gestão do transporte na coleta de resíduos sólidos urbanos

\section{Carla Claudia Apacani Fernández carlisss_3@hotmail.com \\ ORCID 0000-0003-1320-0055 \\ Universidad Privada Domingo Savio, Bolivia}

Artículo recibido mayo 2020 | Arbitrado en julio 2020 | Publicado en septiembre 2020

\section{RESUMEN}

El propósito de la investigación es el desarrollo de una aplicación Web para para fortalecer la gestión del transporte en la recolección de residuos sólidos urbanos de la Empresa Municipal de Aseo Potosí (EMAP). Se parte de un diagnóstico de las necesidades de los usuarios y prestadores de servicio. Se estableció como objeto de estudio al sistema de gestión del transporte en esta empresa. La investigación es proyectiva tecnológica, el diseño es de campo. Las técnicas de recolección de datos son la observación, la encuesta y la entrevista y los instrumentos la guía de observación, el cuestionario y el guion de entrevista. Como resultado se presenta la aplicación Web para la gestión del transporte en EMAP que se construyó aplicando la metodología de gestión de proyectos SCRUM que permite dar respuesta a las necesidades de la empresa.

Palabras clave: Aplicación web; propuesta tecnológica; gestión de transporte; Empresa Municipal de Aseo Potosí; recolección de residuos sólidos; SCRUM

ABSTRACT

RESUMO

The purpose of the research is the development of a Web application to strengthen the management of transportation in the collection of solid urban waste of the Municipal Company of Potosí Cleaning (EMAP). It starts from a diagnosis of the needs of users and service providers. The transport management system in this company was established as an object of study. Research is technological projective, design is field. The data collection techniques are the observation, the survey and the interview and the instruments the observation guide, the questionnaire and the interview script. As a result, the Web application for transport management in EMAP is presented, which was built by applying the SCRUM project management methodology that allows responding to the needs of the company.

Key words: Web application; technological proposal; transportation management; Municipal Cleaning Company Potosí; solid waste collection; SCRUM
The purpose of the research is the development of a Web application to strengthen the management of transportation in the collection of solid urban waste of the Municipal Company of Potosí Cleaning (EMAP). It starts from a diagnosis of the needs of users and service providers. The transport management system in this company was established as an object of study. Research is technological projective, design is field. The data collection techniques are the observation, the survey and the interview and the instruments the observation guide, the questionnaire and the interview script. As a result, the Web application for transport management in EMAP is presented, which was built by applying the SCRUM project management methodology that allows responding to the needs of the company.

Key words: Web application; technological proposal; transportation management; Municipal Company of Potosí Cleaning; solid waste collection; SCRUM 


\section{INTRODUCCIÓN}

La Empresa Municipal de Aseo Potosí (EMAP) gestiona de forma integral el aseo urbano, para lo cual planifica, organiza, dirige y controla los procesos inherentes al transporte de los desechos sólidos. Los tipos de transporte que presta son recolección domiciliaria, recolección auxiliar y recolección por contrato y barrido. Este servicio de recolección generalmente es rutinario, en el sentido que las rutas están preestablecidas, sin embargo temporalmente pueden presentar alteraciones para cumplir con exigencias fuera de la ruta para atender a: lugares con acumulaciones de residuos generados por el servicio de barrido, sectores que presenten un significativo crecimiento de residuos diario, calles no contempladas dentro del recorrido del carro basurero y zonas de relevancia turística donde las acumulaciones de basura inician fuera del horario del servicio. Cuando este servicio lo prestan unidades recolectoras de la empresa, que ya han terminado su turno de trabajo, se denomina Servicio de Recolección Auxiliar (SRA), cuyo objetivo es evitar la propagación de focos de contaminación. El SRA presenta las siguientes etapas:

Proceso de identificación de puntos claves. Esto se realiza mediante el recorrido de varias zonas de Potosí, por el supervisor y el técnico representante, se presta especial atención a los lugares históricos y turísticos de la ciudad. También considera las denuncias de los usuarios de forma personal, a través de las redes sociales o medios de comunicación.

Comunicación con el conductor de la unidad asignada. Esta etapa presenta problemas de logística porque la información no se gestiona de manera adecuada y en ocasiones acuden más de una unidad al punto de recolección.
Los procesos para gestionar la información del SRA poseen una deficiente comunicación, esto ocasiona retrasos, pérdida de recursos materiales y desgaste del personal. Para hacer que el desarrollo de operaciones se realice de la forma más eficiente se plantea una propuesta tecnológica para optimizar la comunicación de las diferentes operaciones y al centralizar la información de todos los procesos y lograr una mejor operatividad y calidad del servicio.

Por consiguiente la investigación se plantea como interrogante ¿Cuáles son las características de una propuesta tecnológica basada en un sistema de información que permita optimizar la gestión del de recolección y transporte de residuos sólidos por la EMAP en la ciudad de Potosí, Bolivia?

El objetivo que guía el proceso investigativo es desarrollar una Aplicación web basada en un sistema de información para fortalecer la gestión del transporte en la recolección de residuos sólidos urbanos de la empresa EMAP de la ciudad de Potosí, Bolivia.

\section{MÉTODO}

Se trató de una investigación proyectiva de tipo tecnológica, ya que se genera una propuesta para atender los resultados del diagnóstico de necesidades. Las técnicas utilizadas para la recolección de los datos son la entrevista, la encuesta y la observación, en relación los instrumentos quedaron constituidos por un guion de entrevista, un cuestionario, una guía de observación y una lista de cotejo. El diseño es de campo ya que se recolectan los datos en la empresa y en la ciudad. La población estuvo conformada por diez trabajadores, cuatro supervisores y un administrador para un total de 15 personas.

Las variables de la investigación se resumen en el Cuadro 1. 
Cuadro 1. Operacionalización de variables

\begin{tabular}{|c|c|c|c|c|}
\hline $\begin{array}{c}\text { Variable } \\
\text { Dependiente }\end{array}$ & $\begin{array}{l}\text { Definición } \\
\text { Conceptual }\end{array}$ & $\begin{array}{l}\text { Definición } \\
\text { Operacional }\end{array}$ & Dimensiones & Indicadores \\
\hline $\begin{array}{l}\text { Sistema de } \\
\text { Gestión de } \\
\text { transporte }\end{array}$ & $\begin{array}{l}\text { Es una } \\
\text { herramienta } \\
\text { desarrollada } \\
\text { que recoge, } \\
\text { almacena, } \\
\text { procesa y } \\
\text { dispone } \\
\text { información } \\
\text { relacionada } \\
\text { con las } \\
\text { operaciones } \\
\text { de } \\
\text { transporte } \\
\text { de } \\
\text { mercancías } \\
\text { en una } \\
\text { organización. }\end{array}$ & $\begin{array}{l}\text { Se sirve de un } \\
\text { conjunto de } \\
\text { elementos que } \\
\text { administran la } \\
\text { información } \\
\text { de fuentes } \\
\text { externas e } \\
\text { internas } \\
\text { relacionadas } \\
\text { con las } \\
\text { operaciones } \\
\text { de traslado de } \\
\text { materiales de } \\
\text { una } \\
\text { organización a } \\
\text { través de } \\
\text { modos de } \\
\text { transporte, } \\
\text { valiéndose a } \\
\text { su vez de la } \\
\text { integración de } \\
\text { procesos de } \\
\text { áreasalternas } \\
\text { que } \\
\text { complementan } \\
\text { la } \\
\text { funcionalidad } \\
\text { que } \\
\text { se tiene por } \\
\text { metacumplir. }\end{array}$ & $\begin{array}{l}\text { Logística y } \\
\text { planificación }\end{array}$ & $\begin{array}{l}\text { - Administración } \\
\text { de información } \\
\text { - Tiempo de } \\
\text { respuesta } \\
\text { - Proceso de } \\
\text { reclamo } \\
\text { - Tiempo de } \\
\text { transporte } \\
\text { - Técnicas de } \\
\text { monitoreo } \\
\text { - Control de } \\
\text { transporte } \\
\text { - Herramientas } \\
\text { para la } \\
\text { planificación } \\
\text { - Asignación de } \\
\text { personal } \\
\text { - Asignación de } \\
\text { movilidades } \\
\text { - Plan de rutas } \\
\text { - Plan de } \\
\text { mantenimiento }\end{array}$ \\
\hline
\end{tabular}

RESULTADOS

Los resultados de la entrevista, todos los entrevistados conocen la razón de ser de la EMAP, cumple con las actividades de limpieza, recolección, transporte y disposición final de los residuos sólidos urbanos de la ciudad de Potosí. De manera general los servicios que la empresa presta al cliente la empresa son: barrido, recolección domiciliaria, recolección de residuos especiales.
El servicio de transporte de recolección de residuos urbanos de la empresa ya cuenta con rutas definidas, a excepción de algunos recorridos del transporte auxiliar, la empresa alcanza a cubrir el 60\% del área de la ciudad, la razón a del porcentaje restante es porque se encuentra ubicado en las periferias de la ciudad, lugares a los que no es posible llegar, ya sea por el tamaño de las movilidades o por la cantidad de estas. 
La identificación los puntos de parada del transporte de recolección se realizan de la manera que se describirá a continuación: Para el servicio de recolección domiciliario las paradas que realizan los carros basureros en las calles son de esquina a esquina. Para identificar los puntos a cubrir generados por el servicio de barrido o los que se ha llamado puntos clave para distinguirlos mejor. Se cuenta con cuatro supervisores, tres de ellos supervisan las zonas de barrido de las señoras, el cuarto supervisor se encarga de evidenciar si existe basura en algunos sitios de la ciudad. Al encontrarse repartidos en diferentes sectores de la ciudad los cuatro supervisores se comunican por radio base para informar en qué lugar hay basura de manera inmediata se dirige la movilidad de emergencia a recoger dichos residuos.

Los recursos humanos o el personal que interviene en la operación de transporte consta de: para realizar el servicio de recolección y conducir el carro basurero se cuenta con un mínimo de tres personas por cada carro basurero, cuatro si el número de trabajadores aumenta. Para el mantenimiento de las movilidades la empresa tiene un mecánico que revisa el estado de todos los carros basureros.

La administración de la información para gestionar el transporte se lo almacena en carpetas de documentos. En lo que respecta a la designación de rutas se tiene una planilla de control de la salida de los carros basureros.

La planificación de las rutas del transporte se establece en consenso con el jefe del departamento y los supervisores, observando que está claramente definida, estas rutas no sufren cambios continuos ni permanentes, debido a que son conocidas por los conductores, los clientes y la empresa misma.

$\mathrm{Si}$ se cuentan con sistemas de seguimiento y localización de las movilidades. Desde hace tres meses atrás se instaló tecnología GPS a las movilidades, las cuales se monitorean gracias al sistema MONET, optando por el paquete de monitoreo de vehículos básico.

Los recursos para la operación del transporte suelen ser insuficientes en ocasiones, el gasto que representan estos recursos está en función al Presupuesto por Objetivos Alcanzados, que se genera cada año, dando un límite del costo que no se debe sobrepasar. En lo que respecta al transporte de material de desechos se intenta abarcar todas las zonas posibles, sin embargo también se cometen errores al recorrer las mismas rutas o no completar toda la ruta que se debe realizar en el servicio domiciliario.

La empresa atiende someramente la relación con el cliente, primeramente se les orienta a los trabajadores para que tengan una buena relación humana con el consumidor del servicio, para proveer una adecuada atención al momento de recolectar los residuos domiciliarios. No se provee información oficial de las rutas del recorrido domiciliario sino que se espera que sean aprendidas empíricamente, tampoco se proporciona información de la ubicación de contenedores de basura.

Como resultado de la observación: No existe medición de indicadores que proveen información sobre el desempeño del transporte al momento de la recolección de residuos. Dependiendo a diversas situaciones en ocasiones no se cubre toda la ruta que tradicionalmente se sigue, de esto no tiene conocimiento el cliente. Las rutas 
son predeterminadas y rotan de movilidad y chofer cada tres meses al igual que los sectores de barrido con los grupos de barrido. Las rutas sufren alteraciones temporales en caso de la existencia de alguna calle bloqueada $\mathrm{u}$ otro inconveniente. Se suele llegar a los lugares establecidos dentro del horario que se ha creado según el recorrido.

Existen esquinas que no son atendidas por el servicio de recolección domiciliario aunque se pueda acceder a estos lugares, sin embargo son atendidos por el servicio de recolección auxiliar a tempranas horas de la mañana periódicamente. En la noche es cuando se presencia más basura en los alrededores de la zona central de la ciudad. Existen puntos recurrentes de acumulación de basura.

Los resultados del cuestionario se resumen: (a) la identificación de los puntos de parada del transporte para recoger el material se realiza por acuerdos con el cliente $(100 \%)$, también se opta por tener un recorrido predefinido $(86,67 \%)$ por el servicio que se ofrece.

Los indicadores más frecuentes para medir $\mathrm{y}$ en consecuencia conocer el desempeño del servicio de transporte, de mayor a menor son: la puntualidad (100\%), tiempo de viaje $(100 \%)$, costo $(100 \%)$, la velocidad $(86,67 \%)$, la demanda $(86,67 \%)$ y la seguridad de carga $(66,67 \%)$.

Las herramientas que emplean para el seguimiento y monitoreo de movilidades, son: El teléfono móvil (73,3\%), el GPS (66.7\%) y el cronómetro $(26,66 \%)$.

Los indicadores más frecuentes para definir el coste en la gestión de transporte, son: el consumo de combustible (100\%), Costos de Mantenimiento (100\%).

\section{Propuesta}

La propuesta es un Sistema de Información Gerencial (SIG), al cual consiste en la integración del trabajo de la informática, la ciencia de la administración y la investigación de operaciones con una orientación práctica hacia el desarrollo de soluciones de sistemas para los problemas del mundo real y la administración de los recursos de tecnología de la información (Laudon y Laudon, 2012). No solo es un programa, la información gerencial implica esencialmente la comunicación entre las personas sobre aquellos eventos que afectan el trabajo que desempeña su institución (Waterfield y Ramsing, 1998).

La EMAP es una empresa de servicios de transporte que realiza en esencia destacar el movimiento físico de los desechos y finalmente se disponen en el relleno o vertedero. El proceso del transporte tiene tres principios: la velocidad, la consistencia y el control tomando en cuenta estos principios se deben tomar acciones para reducir costos optimizando las rutas y atendiendo la satisfacción del cliente.

La aplicación Web tiene las siguientes características:

1. Centraliza la información para gestionar sistematización de los procesos la gestión de la recolección terrestre de desechos sólidos.

2. Reduce el tiempo y el uso de recursos.

3. Funciona como mínimo en dos tipos de plataforma celulares y computadoras.

4. Genera mapas que permitan observar de forma gráfica el monitoreo de transporte, las rutas domiciliarias, la distribución de puntos de parada 
independientes a las rutas y la ubicación de contenedores.

5. Se utilizaron las herramientas Open Street Maps y Leaflet ya que ofrecen licencia abierta a base de datos $y$ permite la edición.

6. Fortalece la gestión de: la información de origen interno y externo, el desempeño de los conductores, recolección domiciliaria, recolección auxiliar, programación para el servicio del barrido, programación y trazado de rutas.

7. Genera documentos que funcionan como reportes para el monitoreo y la evaluación de los procesos diferenciados por zonas, rutas y tipos de servicio.

Las herramientas utilizadas para desarrollar la aplicación web, la cual será principalmente utilizada en ordenadores y para el cliente también en dispositivos móviles, son:

\section{Entorno de Ejecución Node.js}

Concebido como un entorno de ejecución de JavaScript orientado a eventos asíncronos, Node está diseñado para construir aplicaciones en red escalables. Node lleva el modelo de eventos un poco más allá, este presenta un bucle de eventos como un entorno en vez de una librería. (Node.js Foundation, s.f.)

Node.js permite la ejecución de JavaScript en el servidor, lenguaje antes limitado al lado del cliente. Este entorno del lado del servidor (basado en eventos) utiliza el motor V8 (desarrollado por google) para ejecutar JavaScript a gran velocidad. Esto último se debe a que V8 compila JavaScript en código de máquina nativo, en lugar de interpretarlo o ejecutarlo.
Por diversas razones como su rapidez, ligereza, escalabilidad, además de ofrecer otras herramientas que ayudan al desarrollo, se utiliza este entorno para hacer la aplicación web.

\section{JavaScript}

También conocido por su abreviación JS, es un lenguaje ligero e interpretado, orientado a objetos con funciones de primera clase, más conocido como el lenguaje de script para páginas web, pero también usado en muchos entornos sin navegador, tales como node.js, Apache CouchDB y Adobe Acrobat. Es un lenguaje script multi-paradigma, basado en prototipos, dinámico, soporta estilos de programación funcional, orientada a objetos e imperativa. (Grupo de colaboradores de MDN Web Docs, 2018).

JavaScript tiene la capacidad de integrarse perfectamente con el sistema operativo al igual que con gran parte de navegadores web. Es el lenguaje de programación utilizado por el entorno de ejecución Node.js y gracias a este ha sido llevado a otro nivel, trabaja en la programación de aplicaciones web, alcanzando su funcionalidad tanto en el front- end para agregar dinamismo como en el back-end por medio de Node.

\section{Express: Infraestructura de} aplicaciones web Node.js

Express es una infraestructura web rápida, minimalista y flexible para las aplicaciones Node.js. En general, se prefiere Express a Express.js, aunque está también se acepta (Node.js Foundation, 2017). Ayuda a no comenzar necesariamente desde cero, como todo framework, sino que provee una infraestructura que permite agilizar el desarrollo de la aplicación web. 
Express es propio de Node, por lo cual se posiciona como el primero en la lista de frameworks a usar, además provee plugins de alto rendimiento nombrados middleware. Middleware permite la interacción o comunicación entre aplicaciones, o bien entre la aplicación y otro software, red, hardware y/o sistema operativo.

\section{Bootstrap: Framework Front-end}

Bootstrap se encuentra compuesto por un conjunto de herramientas de código abierto para desarrollar con HTML, CSS y JS. Cubre el área front-end de desarrollo de aplicaciones web, se basa en el diseño responsive el cual permite adaptabilidad tanto en ordenadores como dispositivos móviles, agiliza el desarrollo debido a que provee recursos prediseñados y no impide la implementación de otras plantillas CSS. Bootstrap básicamente es: Un framework desarrollado y liberado por Twitter que tiene como objetivo facilitar el diseño web. Permite crear de forma sencilla webs de diseño adaptable, es decir, que se ajusten a cualquier dispositivo y tamaño de pantalla y siempre se vean igual de bien. Es Open Source o código abierto, por lo que se puede usar de forma gratuita y sin restricciones. (Punto Abierto S. Coop. Mad., 2009-2017)

\section{HTML5}

Cuyas sigla significa en español Lenguaje de Marcado para Hipertextos (HyperText Markup Language)"es el elemento de construcción más básico de una página web y se usa para crear y representar visualmente una página web. Determina el contenido de la página web, pero no su funcionalidad" (Grupo de colaboradores de MDN Web Docs, 2017).
Para estructurar la presentación de la aplicación web se recure al lenguaje de marcado HTML, debido a su sencillez y popularidad, en provecho de este último existen más herramientas que hacen uso de este lenguaje de marcado, como Bootstrap.

\section{CSS (Hojas de Estilo en Cascada):}

Al igual que HTML CSS no es realmente un lenguaje de programación. Es un lenguaje de hojas de estilo, es decir, te permite aplicar estilos de manera selectiva a elementos en documentos HTML (Grupo de colaboradores de MDN Web Docs, 2018). CSS es una herramienta que permite la personalización del diseño de las aplicaciones web, para contribuir al que la plataforma logre ser comprendida de forma más fácil y rápida para el usuario.

Leaflet: es la librería open source líder de JavaScript para la publicación de mapas interactivos y aptos para dispositivos móviles (Agafonkin, 2018). La herramienta Leaflet está diseñada de manera que toma en cuenta la simplicidad, el rendimiento y facilidad de uso. Puede extender sus funciones debido a sus muchos complementos, posee bastante documentación, y un código fuente simple y legible. Funciona adecuadamente en distintas plataformas.

Open Street Maps: es al mundo de los mapas es lo que la Wikipedia al mundo de la enciclopedia. Se trata de una plataforma libre y colaborativa (Genbeta, 2012). Estos obtienen información por la contribución de sus usuarios. A diferencia de Google Maps y por su tipo de licencia no tiene límite de 25.000 peticiones a sus servidores.

Angular: AngularJS es $100 \%$ JavaScript, $100 \%$ del lado del cliente y compatible con navegadores de escritorio y móviles. Así que definitivamente no es un 
complemento o alguna otra extensión del navegador nativo" (Angular, s.f.). Angular brinda la vista dinámica que HTML, CSS o JavaScript que no puede dar a los usuarios.

Visual Studio Code: es un editor de código redefinido y optimizado para construir y depurar modernas aplicaciones web y de la nube. Visual Studio Code es libre y está disponible en su plataforma favorita: Linux, Mac OSX y Windows (De Oliveira, Polat, y Belkheraz, 2018, p. 614). Algunas ventajas de Visual Studio Code son la integración de un terminal direccionado por defecto en la misma carpeta donde se ubica el proyecto, a diferencia de otros editores de Microsoft ofrece un entorno de desarrollo simplificado permitiendo editar el código sin requerir compilación continua.

MySQL: Base de datos es un sistema de gestión de base de datos relacional (RDBMS) de código abierto, basado en lenguaje de consulta estructurado (SQL). (TechTarget, S.A, 2005-2018) sirve para la gestión de bases de datos relacionales, las cuales hacen uso de tablas y esquemas. Es importante mencionar que MySQL cuenta con una licencia de funcionamiento GPL, el uso de Mysql es frecuente en las empresas, también provee interfaces gráficas que ayudan a su utilización.

Las herramientas para el desarrollo de la aplicación Móvil son:

Android Studio y herramientas compatibles, Android Studio es el entorno de desarrollo integrado (IDE) oficial para el desarrollo de aplicaciones para Android y se basa en Intellij IDEA (Conoce Android Studio: Developer Android, 2018). Android Studio, además de poseer un potente editor de códigos y proporcionar herramientas para el desarrollo, también ofrecen más funciones que mejoran la productividad durante la compilación de aplicaciones para
Android, algunas de estas son: sistema de compilación basado en Gradle flexible, emulador rápido con varias funciones, entorno unificado en el que puedes realizar desarrollos para todos los dispositivos Android, Instant Run para aplicar cambios mientras tu app se ejecuta sin la necesidad de compilar un nuevo APK, los lenguajes de programación que principalmente maneja son Kotlin y JAVA.

Api REST es un tipo de arquitectura de desarrollo web que se apoya totalmente en el estándar HTTP" (Marqués, 2013). REST significa "Transferencia de Estado Representacional, permite a la aplicación móvil obtener datos sin la interfaz de una aplicación web o también ejecutar alguna acción sobre estos, ofreciendo métodos de autenticación y validación.

Draw.io es un componente tecnológico de código abierto para la elaboración de aplicaciones de creación de diagramas y la aplicación de creación de diagramas para usuarios finales (Alder $y$ Benson, s.f.).

Arquitectura MVC Modelo Vista Controlador (MVC) es un estilo de arquitectura de software que separa los datos de una aplicación, la interfaz de usuario, y la lógica de control en tres componentes distintos (Universidad de Alicante, s.f.)

El diseño MVC en el desarrollo del proyecto, permite la separación, previniendo la acumulación de código en un mismo lugar lo cual dificulta no solo la búsqueda sino también la solución de errores. Reutiliza código, por ejemplo en las vistas es posible crear plantillas $\mathrm{y}$ sencillamente llamarlas, una vez realizado algún cambio no habrá necesidad de repetirlo en varios archivos más. 


\section{Metodología SCRUM}

El tiempo, tamaño y recursos con los que cuenta el proyecto son determinantes al momento de escoger técnicas para la correcta gestión de proyectos, por estos motivos se escogió la metodología SCRUM. Es una metodología ágil y flexible para gestionar el desarrollo de software, cuyo principal objetivo es maximizar el retorno de la inversión para su empresa (ROI). Se basa en construir primero la funcionalidad de mayor valor para el cliente y en los principios de inspección continua, adaptación, auto- gestión e innovación (Takeuchi, y Nonaka, 1986).

Un SCRUM es un conjunto de trabajos grupales que están al servicio de la obtención de un propósito. La singularidad del procedimiento radica en la forma en que intervienen los diferentes actores del plan, entre otras cosas los dirigentes, los integrantes de segunda y tercera línea e, inclusive, los propios usuarios o destinatarios. Para dejar más claro lo que distingue a SCRUM de los procedimientos habituales de administración se detallar todas sus 4 fases de ejecución:

1. Planteamiento: un emprendimiento gestionado bajo el modelo SCRUM parte de los objetivos que han trazado con anterioridad el cliente y la compañía. Lo primero es fraccionarlo en entregas parciales, de forma que el cliente logre volver a pensar puntos a los que en inicio no prestó la consideración adecuada o que sencillamente desconocía.

2. Lista de tareas: el grupo de trabajo elabora la lista de tareas que debe tomar en cuenta para cada distribución de resultados. Es primordial llevar a cabo una estimación del esfuerzo requerido para, así, fijas plazos proporcionados.

3. Reuniones: lo mejor es que todos los días el conjunto dedique 15 minutos para reunirse y ponerse alerta de la evolución del plan. En estas sesiones, el jefe del emprendimiento (Scrum Master) debe encargarse de que cada integrante cumpla con los trabajos que le fueron asignadas y motivarles para que la eficacia del equipo no descienda.

4. Demostración: una vez ejecutados los trabajos de cada etapa, el conjunto se agrupa con el cliente para exhibir los adelantos que corresponden. El cliente escoge si replantea los elementos del emprendimiento. $\mathrm{Si}$ es requisito, el conjunto asume nuevos compromisos.

5. Retrospectiva: los integrantes del conjunto se reúnen para apreciar el desarrollo de distribución de resultados y analizan los causantes que podrían hacer mejor de cara en el final del desarrollo. La funcionalidad del Scrum Master consiste en remover estos obstáculos.

Los integrantes del SCRUM son: (a) el Product Owner, Tiene por función mejorar y maximizar el valor del producto, siendo la persona con la responsabilidad de administrar el flujo de valor del producto. (b) El Scrum Team equipo tiende a estar compuesto de 3 a 9 expertos dedicados a desarrollar el producto, auto-organizándose y auto-gestionándose para lograr un aumento del programa al final del período de avance, utilizando los elementos del Product Backlog seleccionados (Sprint Backlog) a lo largo del Sprint Planning. (c) los usuarios finales, Es la persona que utilizará un software o un dispositivo hardware una vez haya sido desarrollado, 
comercializado e instalado. El concepto se apoya en la idea del "objetivo final", la cual indica que un producto de software o hardware debe ser útil al consumidor.

Asimismo es importante presentar los artefactos comprendidos por SCRUM: (a) Product Backlog, También llamado Pila de Productos es una lista de los requisitos del usuario, la cual partiendo de una visión inicial del producto tiende a crecer $\mathrm{y}$ evolucionar durante el proceso del desarrollo. (b) Sprint Backlog, Conocido como Pila de Sprint en español. Un Sprint, es el nombre que se le da a cada iteración del desarrollo. Entonces es una lista de los trabajos que el equipo debe realizar durante el sprint para generar el incremento planificado. (c) Burndown Chart, Es utilizada para la medición visual que exhibe el trabajo completado por día, frente a la tasa de finalización proyectada para la versión de hoy del emprendimiento. $\mathrm{Su}$ propósito es aceptar que el emprendimiento esté en el sendero para dar la satisfacción esperada dentro del límite de tiempo esperado. (d) La tasa de avance de un Scrum Team se denomina "velocidad". Luego de unos pocos Sprints, la agilidad de un Scrum Team posiblemente va a ser predecible y dejará una estimación muy precisa sobre el tiempo primordial hasta que se completen todas las entradas en el Registro de productos Scrum.

Las Reuniones son un aspecto muy importante en esta metodología, se dividen en dos partes para responder al ¿Qué? Se fundamenta en el diagnóstico de necesidades para dar respuesta a cada uno de los aspectos negativos encontrados, la segunda parte es el ¿Cómo? se planifican la iteraciones se elabora la táctica para encontrar el resultado más destacable y viable con el mínimo esfuerzo. Esta actividad la ejecuta el equipo ya que es el responsable de ordenar su trabajo, se crea la lista de tareas de la iteración (Sprint Backlog).

A continuación se presentan los diagramas que permitieron el desarrollo de la aplicación Web y las vistas de la aplicación como producto final. 


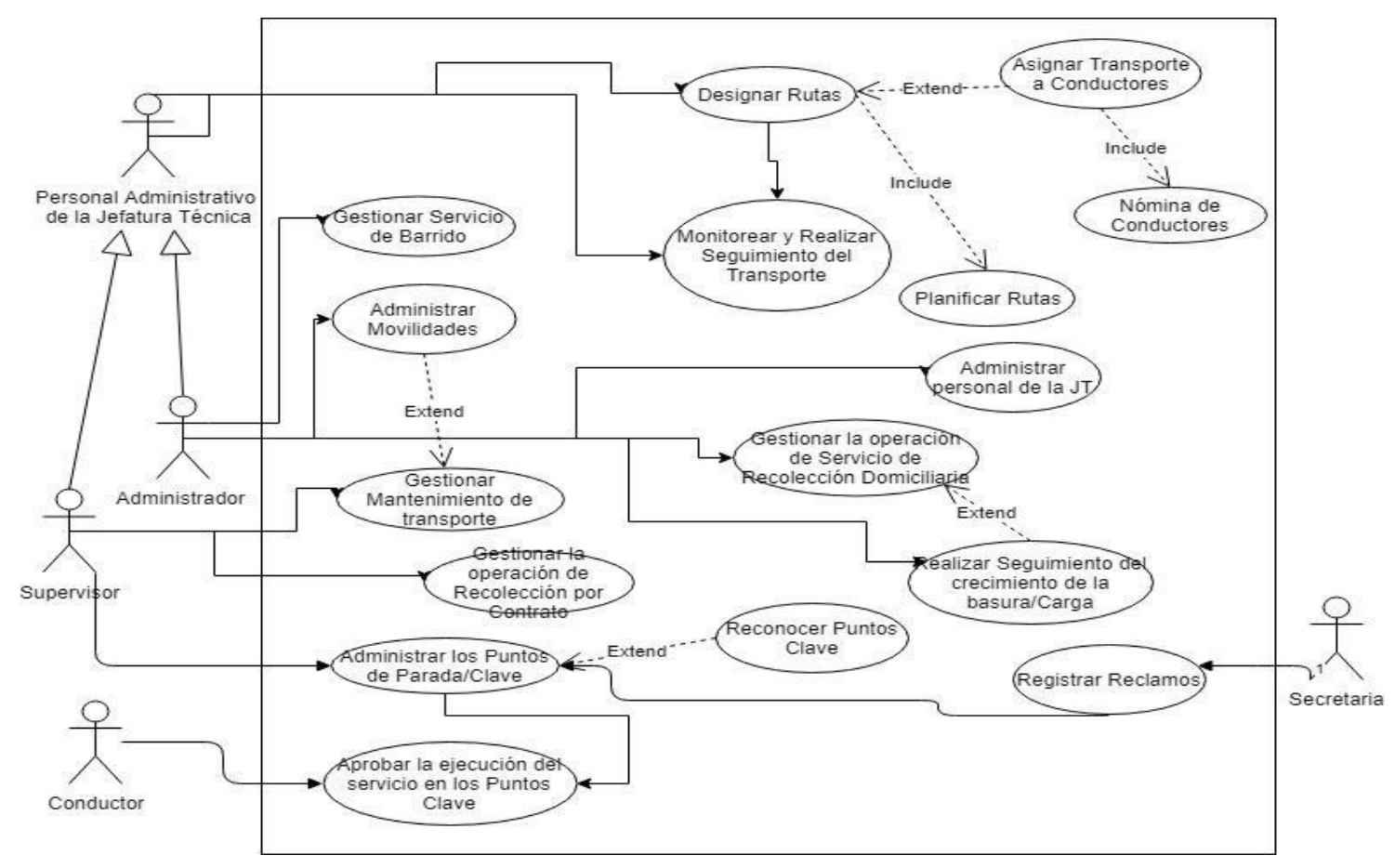

Figura 1. Diagrama de casos de usos de negocios

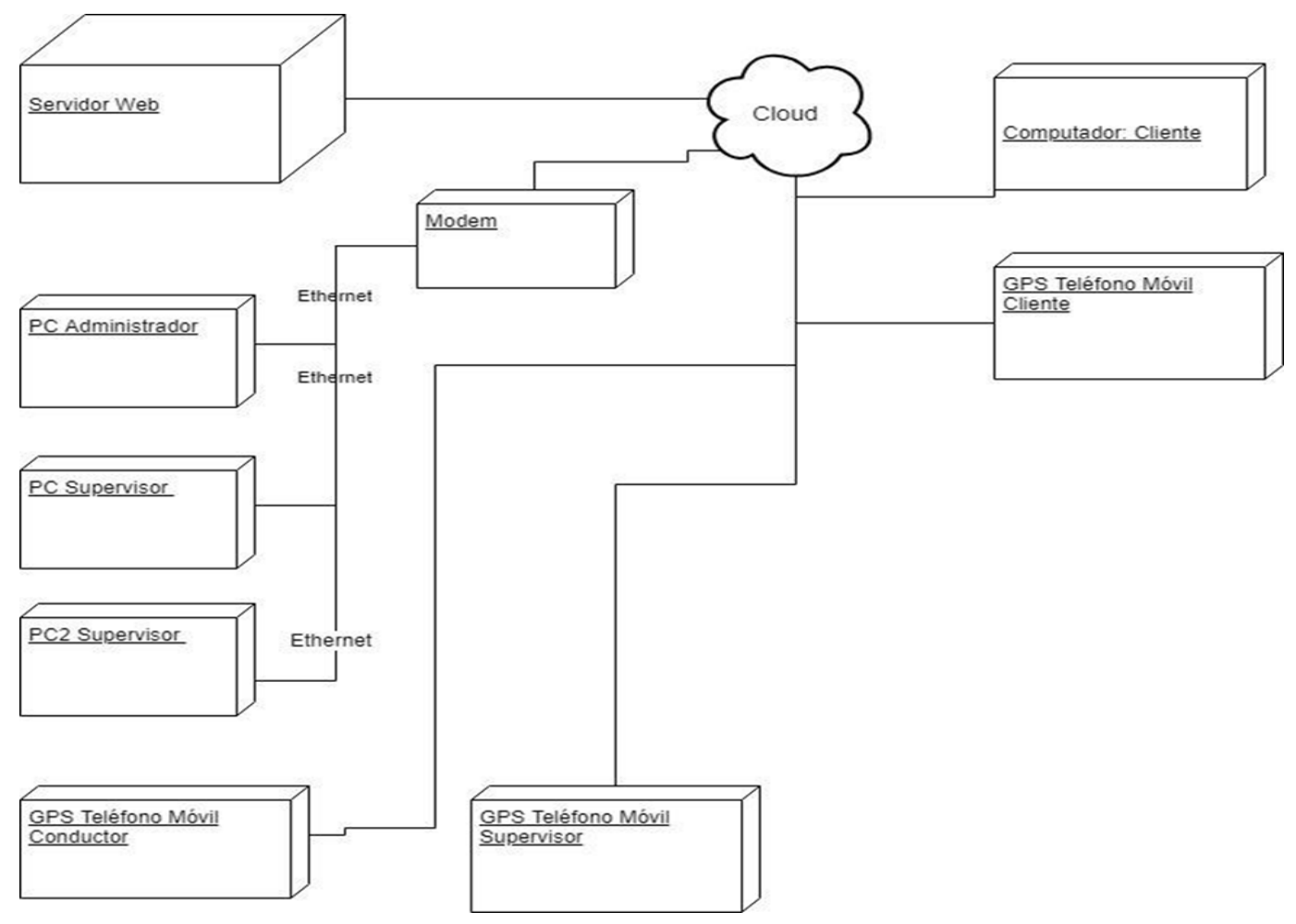

Figura 2. Diagrama de despliegue 


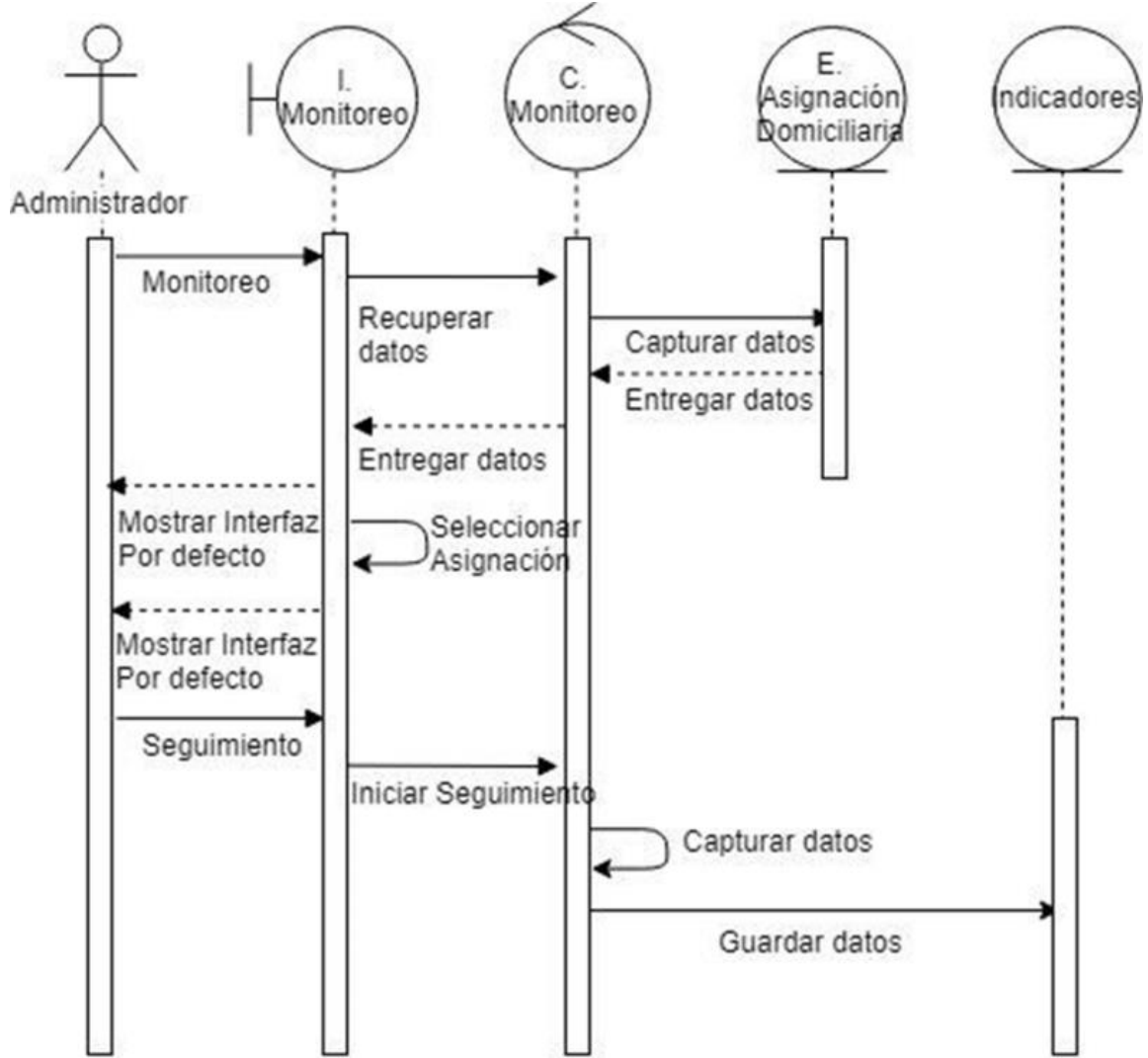

Figura 3. Diagrama de secuencia: Indicadores

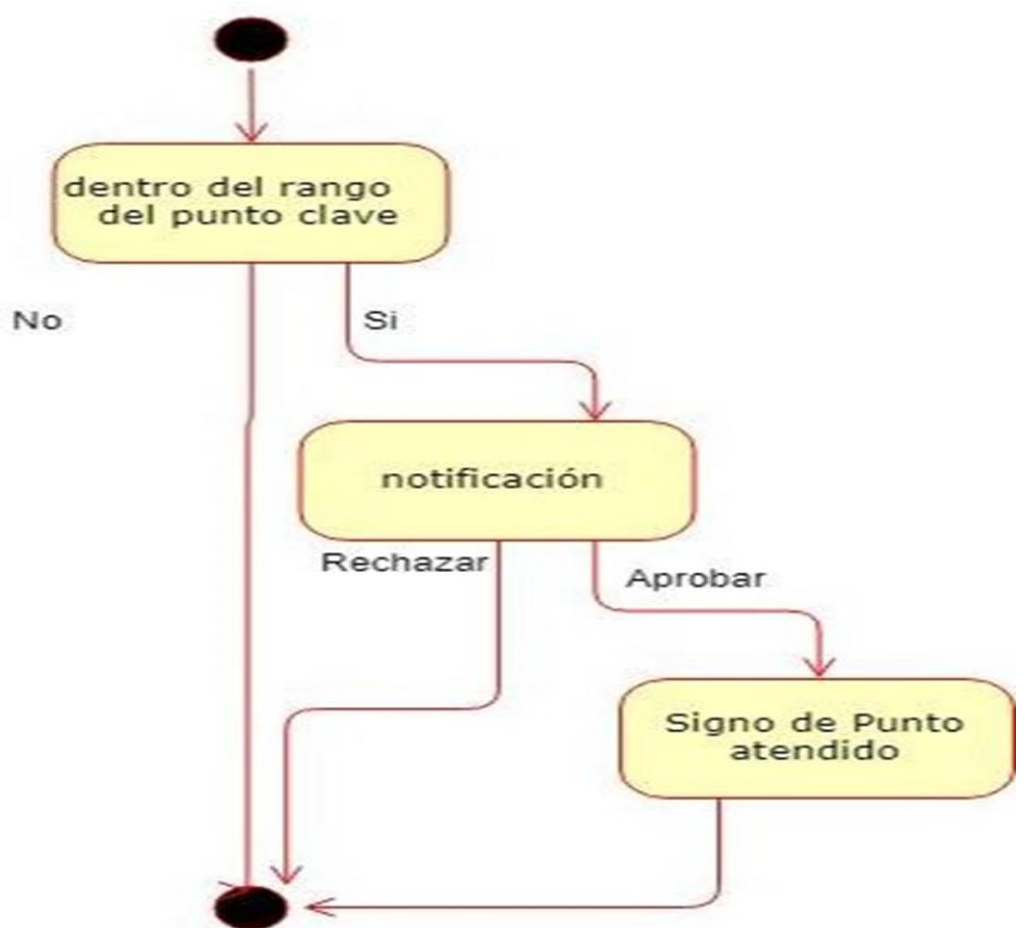

Figura 4. Diagrama de estados: conductores en recolección especial 


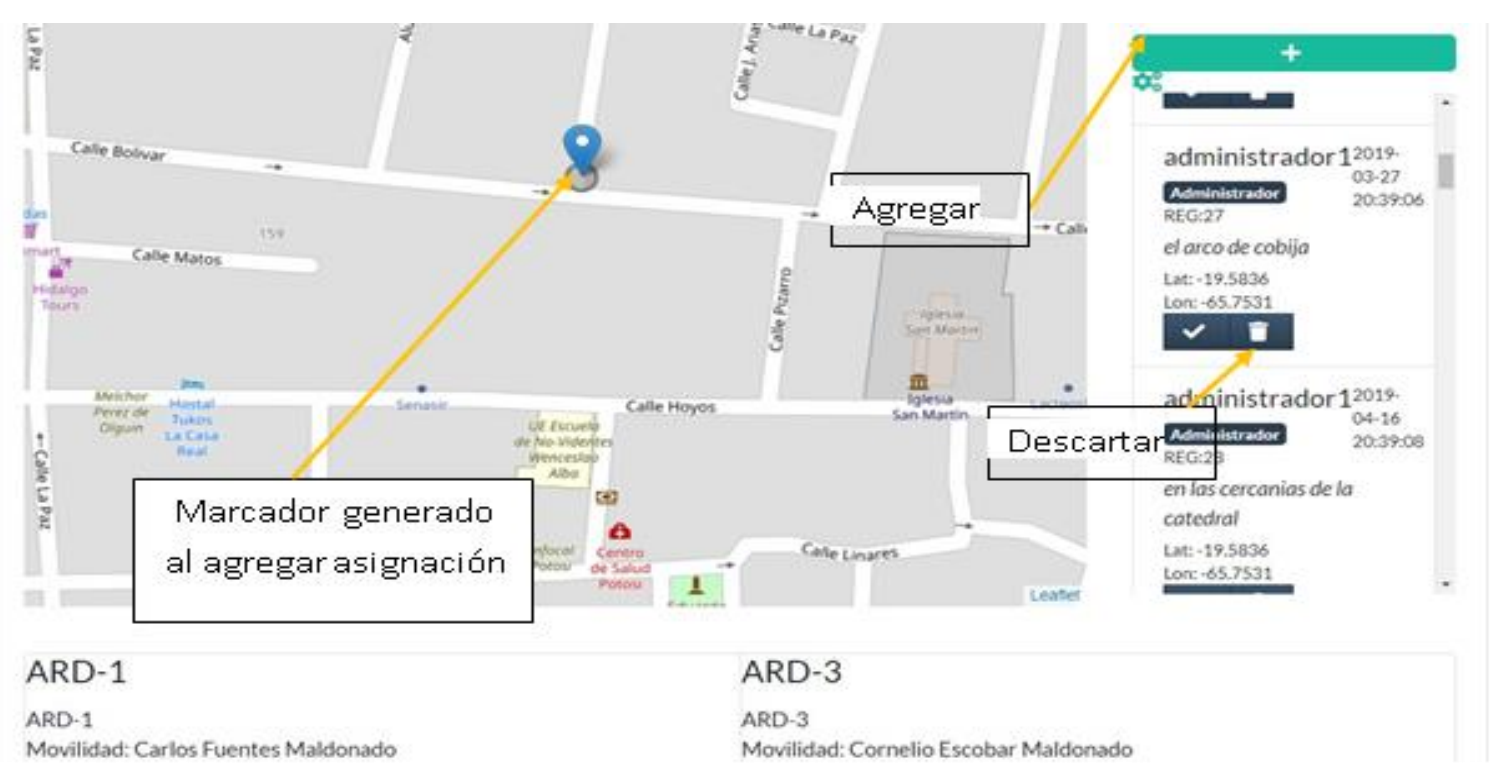

Figura 5. Vista de la aplicación en la asignación de la ruta

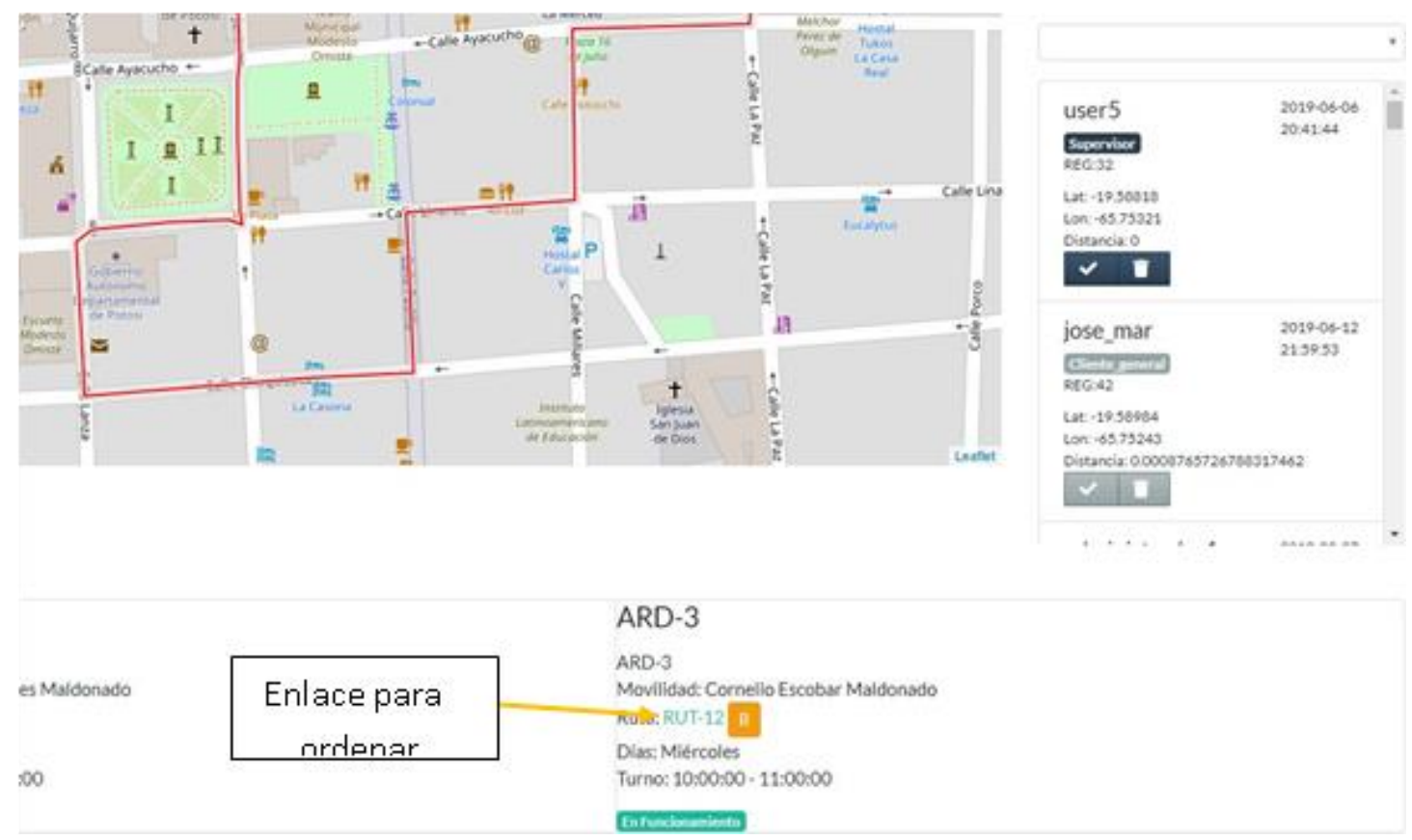

Figura 6. Vista de la aplicación en la opción muestra la ruta y ordena las notificaciones por proximidad a la ruta. 


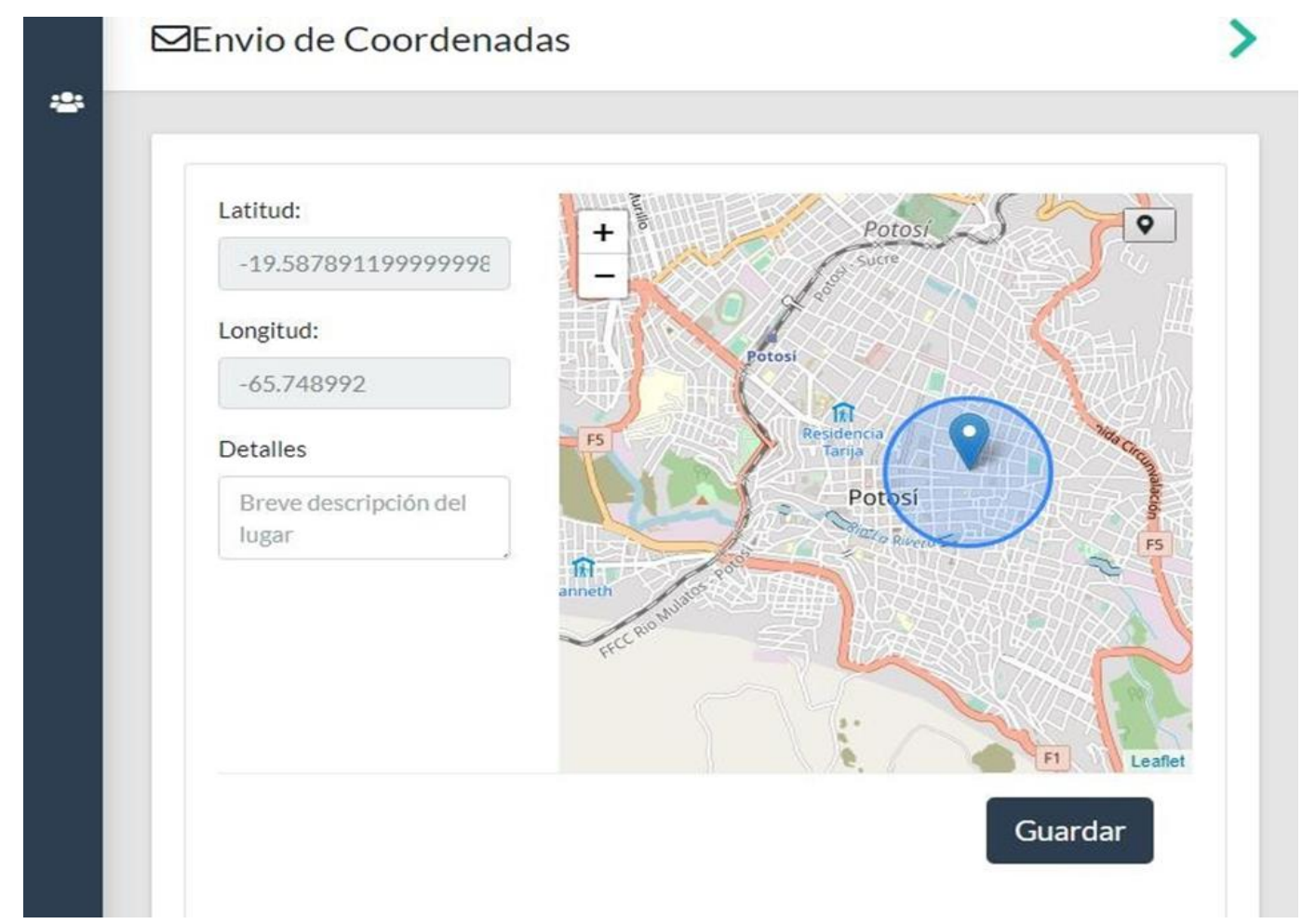

Figura 7. Vista de la aplicación en el envío de coordenadas de localización de la necesidad de servicio.

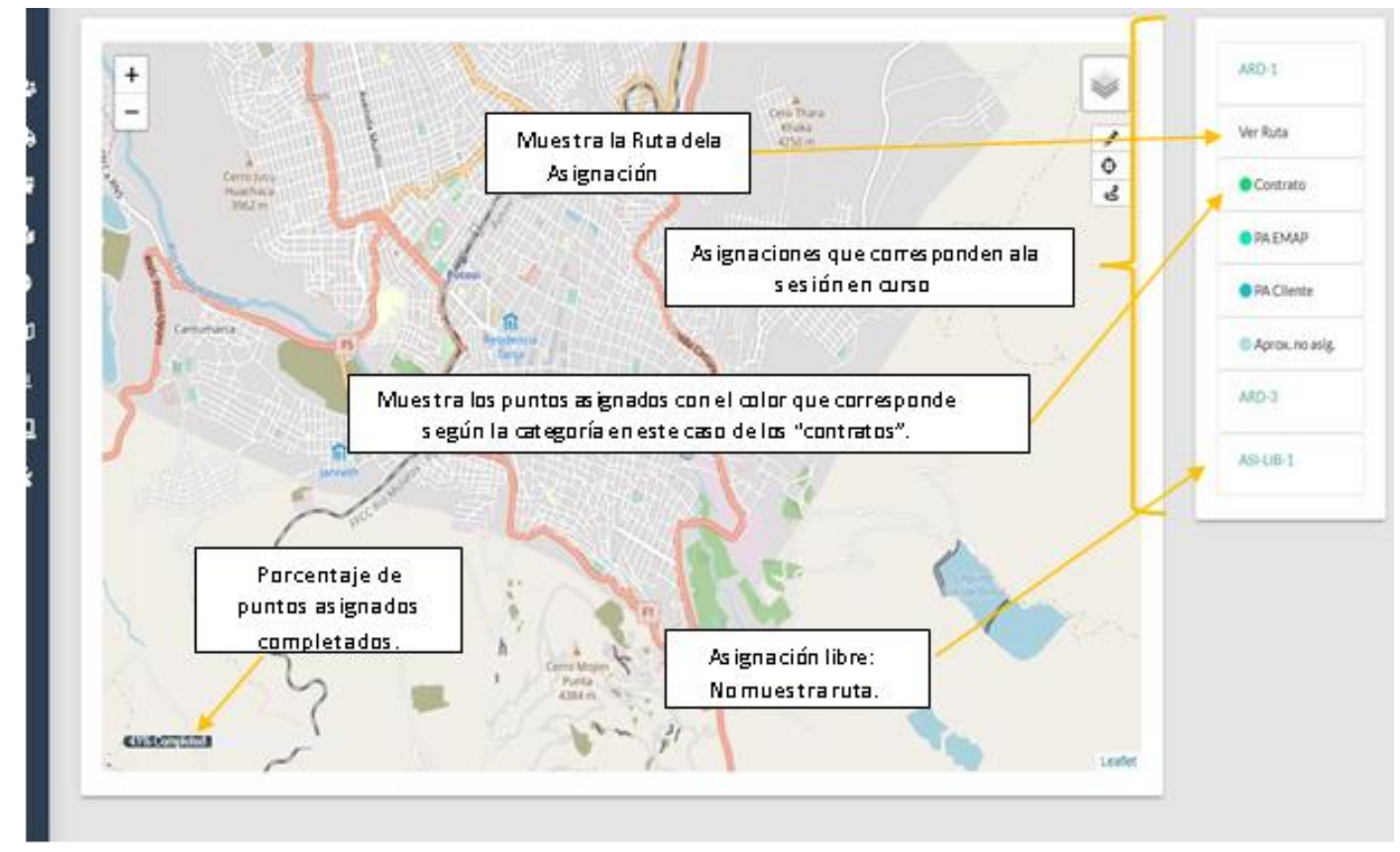

Figura 8. Vista de la aplicación Panel de monitoreo 


\section{CONCLUSIONES}

El diagnóstico del estado actual de la Entidad Municipal de Aseo Potosí. Desde la observación se pudo concluir que el proceso de reclamos de los clientes no se atiende de manera sistemática. No se informa el horario de recolección al cliente, porque éste es variable.

El transportista no comparte información inmediata para conocer las necesidades en el servicio de recolección domiciliario, auxiliar o para residuos especiales, una comunicación inmediata podría beneficiar a la adecuada utilización de recursos.

Se tiene en cuenta al cliente al proporcionar cierta información por medios televisivos y radiofónicos, sin embargo no se promueve su participación en el servicio, ni la empresa se beneficia de la información que puede emitir.

Las rutas predeterminadas sufren cambios temporales, estas rutas rotan de movilidad y conductor cada ciclo ya establecido por la empresa. Este suceso también se efectúa en el servicio de barrido. El sistema Monet se limita al monitoreo de los vehículos. El transporte de desechos sólidos de la EMAP no es eficaz.

\section{REFERENCIAS}

Agafonkin, V. (2018). Leaflet. Disponible: Sitio Web de Leaflet: https://leafletjs.com/

Alder, G., y Benson, D. (s.f.). Sobre Nosotros: Sitio Web draw.io. Disponible: Sitio Web draw.io: https://about.draw.io/aboutus/

Angular. (s.f.). Preguntas Frecuentes: Angular. Disponible: Sitio Web Angular: https://docs.angularjs.org/misc/faq\#isangularjs-a-library-framework-pluginor-a- browser-extension-

Conoce Android Studio: Developer Android. (2018). Disponible: Sitio Web de
Developer Android: https://developer.android.com/studio/i ntro/?hl=es-419

Genbeta (2012). OpenStreetMaps vs. Google Maps: Genbeta. Disponible: Web Genbeta:

https://www.genbeta.com/web/openstr eetmaps-vs-google- maps-quien-gana-yquien-pierde-con-los-ultimos-

movimientos-entorno-a-los- mapas

Grupo de colaboradores de MDN Web Docs. (2017) HTML: MND Web Docs. Disponible: Sitio web de MND Web Docs: https://developer.mozilla.org/es/docs/ Web/HTML

Grupo de colaboradores de MDN Web Docs. (2018). HTML: MND Web Docs. Disponible: Sitio web de MND Web Docs: https://developer.mozilla.org/es/docs/ Web/HTML

Grupo de colaboradores de MDN Web Docs. (2018) CSS básico: MDN Web Docs. Disponible: Sitio Web de MDN Web Docs: https://developer.mozilla.org/es/docs/ Learn/Getting_started_with_the_web/CS S_basics

Grupo de colaboradores de MDN Web Docs. (2018) JavaScript: MDN Web Docs. Disponible: Sitio Web de MDN Web Docs: https://developer.mozilla.org/es/docs/ Web/JavaScript

Marqués, A. (2013). Conceptos sobre APIs REST: Asier Marqués. Disponible: Sitio Web Asier Marqués: http://asiermarques.com/2013/concept os-sobre- apis-rest/

Node.js Foundation. (2017). Glosario: Express. Disponible: Sitio Web de Express:

https://expressjs.com/es/resources/glo ssary.html

Node.js Foundation. (s.f.). Acerca de Node.js: Node JS. Disponible: Node JS: https://nodejs.org/es/about/

Oliveira, J., Polat, E., y Belkheraz, S. (2018). ASP.NET Core MVC 2.0 Cookbook. Birmingham: Packt Publishing Ltd.

Punto Abierto S. Coop. Mad. (2009-2017). Qué es Bootstrap y cuáles son sus ventajas: Punto Abierto. Disponible: Sitio 
Web de Punto Abierto: https://puntoabierto.net/blog/que-esbootstrap-y-cuales-son-sus-ventajas

Softeng. (s.f.). Metodología Scrum para desarrollo de software - aplicaciones complejas: Soften. Disponible: Sitio Web de Softeng: https://www.softeng.es/eses/empresa/metodologias-detrabajo/metodologia- scrum.html

Takeuchi, H., y Nonaka, I. (1986). The new new product development game. Harvard business review, 64(1), 137146.Laudon, K. C., y Laudon, J. P. (2012). Sistemas de información gerencial. (A. V. Romero Elizondo, Trad.) Atlacomulco: Pearson Educación de México, S.A

TechTarget, S.A. (2005-2018). Guía Esencial: Search Data Center. Disponible: Sitio web de Search Data Center: https://searchdatacenter.techtarget.com /es/guia/Guia-Esencial-Las-bases-dedatos-dan-soporte-a-las-tendencias-deTI

Universidad de Alicante. (s.f.). Universidad de Alicante. Disponible: Sitio Web de la Universidad de Alicante: https://si.ua.es/es/documentacion/aspnet-mvc-3/1- dia/modelo-vistacontrolador-mvc.html

Waterfield, C., y Ramsing, N. (1998). Sistemas de información gerencial para instituciones de micro finanzas. (J. Chaparro, Trad.) Washington: PACT Publications 